\title{
PigLeg: prediction of swine phenotype using machine learning
}

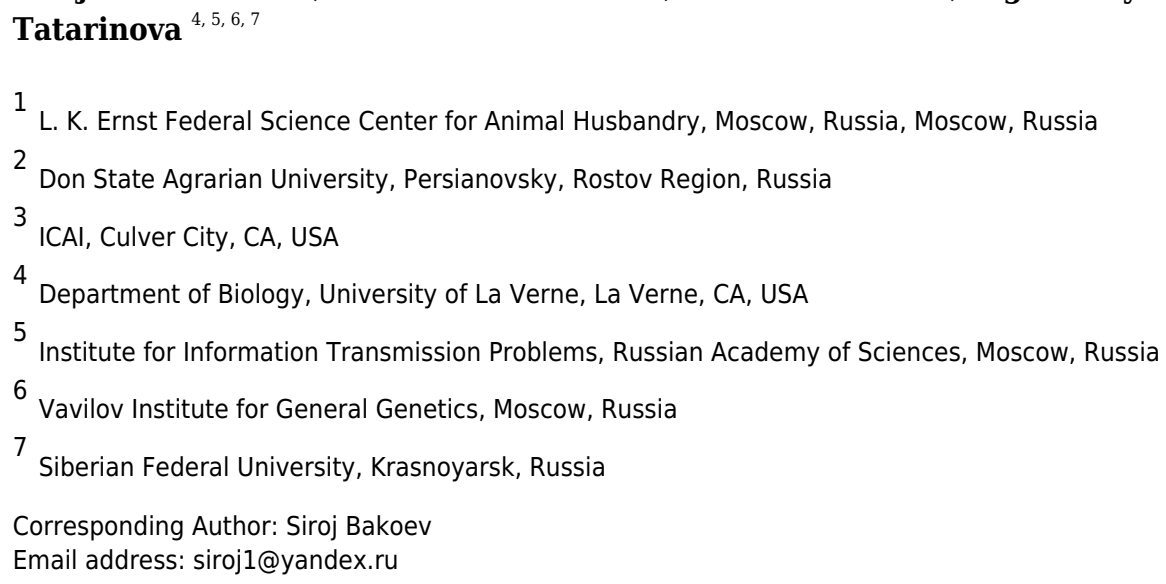

: Industrial pig farming is associated with negative technological pressure on the bodies of pigs. Leg weakness and lameness are the sources of significant economic loss in raising pigs. Therefore, it is important to identify the predictors of limb condition. This work presents assessments of the state of limbs using indicators of growth and meat characteristics of pigs based on machine learning algorithms. We have evaluated and compared the accuracy of prediction for nine ML classification algorithms (Random Forest, K-Nearest Neighbors, Artificial Neural Networks, C50Tree, Support Vector Machines, Naive Bayes, Generalized Linear Models, Boost, and Linear Discriminant Analysis) and have identified the Random Forest and K-Nearest Neighbors as the best-performing algorithms for predicting pig leg weakness using a small set of simple measurements that can be taken at an early stage of animal development. Measurements of Muscle Thickness, Back Fat amount, and Average Daily Gain were found to be significant predictors of the conformation of pig limbs. Our work demonstrates the utility and relative ease of using machine learning algorithms to assess the state of limbs in pigs based on growth rate and meat characteristics. 


\section{PigLeg: prediction of swine phenotype using machine learning}

2 Siroj Bakoev ${ }^{1}$, Lyubov Getmantseva ${ }^{1}$, Maria Kolosova ${ }^{2}$, Olga Kostyunina ${ }^{1}$, Duane Chartier ${ }^{3}$, Tatiana V.

3 Tatarinova ${ }^{4-7}$

$4 \quad{ }^{1}$ L. K. Ernst Federal Science Center for Animal Husbandry, Moscow, Russia; siroj1@yandex.ru

$5 \quad 2$ Don State Agrarian University, Persianovsky, Rostov region, Russia

$6 \quad{ }^{3}$ ICAI, Inc. Culver City, CA, 90230, USA; drc@authentica.org

$7 \quad{ }^{4}$ Department of Biology, University of La Verne, La Verne, CA, 91750, USA; ttatarinova@laverne.edu

$8 \quad{ }^{5}$ The Institute for Information Transmission Problems, Moscow 127051, Russia

96 Vavilov Institute of General Genetics, Moscow 119333, Russia

$10{ }^{7}$ School of Fundamental Biology and Biotechnology, Siberian Federal University, Krasnoyarsk 660041, Russia

$11 *$ Correspondence: siroj1@yandex.ru

12 Received: date; Accepted: date; Published: date

13 Abstract: Industrial pig farming is associated with negative technological pressure on the bodies of pigs.

14 Leg weakness and lameness are the sources of significant economic loss in raising pigs. Therefore, it is 15 important to identify the predictors of limb condition. This work presents assessments of the state of limbs 16 using indicators of growth and meat characteristics of pigs based on machine learning algorithms. We 17 have evaluated and compared the accuracy of prediction for nine ML classification algorithms (Random 18 Forest, K-Nearest Neighbors, Artificial Neural Networks, C50Tree, Support Vector Machines, Naive 19 Bayes, Generalized Linear Models, Boost, and Linear Discriminant Analysis) and have identified the 20 Random Forest and K-Nearest Neighbors as the best-performing algorithms for predicting pig leg 21 weakness using a small set of simple measurements that can be taken at an early stage of animal 22 development. Measurements of Muscle Thickness, Back Fat amount, and Average Daily Gain were found 23 to be significant predictors of the conformation of pig limbs. Our work demonstrates the utility and 24 relative ease of using machine learning algorithms to assess the state of limbs in pigs based on growth 25 rate and meat characteristics.

26 Keywords: Artificial Intelligence, Bioinformatics, Computational Biology, Data Mining \& Machine 27 Learning, Evolutionary Studies, Mathematical Biology, Animal Behavior.

\section{Introduction}

One of the main research tasks in animal husbandry is the discovery of the biological mechanisms influencing animal productivity and finding efficient ways of increasing it. Pork is the most widely 
31 consumed meat in the world. In addition to meat, many valuable products come from pigs: insulin, 32 replacement human heart valves, suede for shoes and clothing, and gelatin for food and industry.

33 Intensive pig farming is associated with negative technological pressure on the development of pigs.

34 Breeding for accelerated development and meatiness leads to a rearrangement of the metabolism in the animal's body, resulting in morphological and functional rearrangements of the internal organs, muscle, adipose, and bone tissues. Changes associated with the cartilage structure are called osteochondrosis (leg weakness). In industrial pig farming, the term "leg weakness" is used to describe the poor constitution of pig legs or the clinical condition associated with lameness or stiffness of movements. Such weakness results from abnormal changes in the cartilage joints and the development of epiphyseal plates, which are responsible for bone enlargement both in length and diameter [1]. Weak epiphyseal plates can break, and the cartilage that covers the joint surface cracks. In the acute phase of the disease, bone fractures may occur near the epiphyseal plate. However, in most cases, the disease takes a chronic form, develops gradually, and manifests itself as incorrect shape and alignment of legs, as well as stiffness of the animal's gait. In this regard, the first step in diagnosing the disease is an exterior assessment of the legs and gait. Typically, pig legs are visually assessed by specially trained personnel using a point system [2].

Rapid advances in next-generation sequencing (NGS) and high-density genotyping technologies allows identification of several quantitative trait loci (QTL) for pig lameness and leg weakness. Leg weakness is partially a heritable trait, with heritability estimates of leg ranging from low $(0.07,[3])$ to moderate $(0.36$, [4]). Despite the agricultural importance of this trait, there has only been a limited number of GWAS for leg weakness. In addition, the trait may be complex and influenced by many factors, such as bone strength, muscle growth, fat accumulation, farming practices, animal activity level, and body weight gain. Therefore, one of the tasks of the present work was to identify these factors using modern statistical approaches.

Rapidly developing data mining approaches are of increasing interest because they provide for acquisition and analysis of information that results in predictive productivity indicators for animals [5-7]. Machine learning (ML) approaches have been successfully used in animal husbandry for early prediction of the growth and quality of adult wool in Australian merino sheep [8], sheep carcass traits from early-life records [9], and skin temperature of piglets [10]. Compared to other statistical approaches, ML is suitable for use even when there are many predictors, missing values, and abnormally distributed data, which is often the case with data obtained from commercial pig production. 
60 In this work, we have evaluated the condition of pig legs by application of ML methods to growth and 61 meat characteristics (see Figure 1). We have compared common ML classification algorithms for predicting 62 the state of the front and hind legs. This led to the identification of the most effective algorithm for 63 predicting leg weakness using a small set of cost-effective and easily measurable sets of functions that can 64 be used in the early period of animal rearing.

\section{Materials and Methods}

66

67

\section{Data sources}

In pig farming, over the past few decades, a primary focus has been on improving meat quality, growth rate, and reproductive qualities of animals. The main parameters for selection are the Average Daily Gain (ADG), Muscle Thickness (MT), and Bacon/Backfat Thickness (BF). In addition, we have investigated other factors that can affect the conformation of legs: breed, year of birth (Birth Date), and gender. The data were obtained from 24,584 pigs of breeds Landrace and Large White. Measurements were made in vivo using ultrasound scanners. ADG is measured in grams, MT and BF are measured in mm (see Table 1).

Front and Back legs were visually assessed using a point system from 1 to 5 (from bad to good). The assessment was performed by specially trained personnel. Points 1 and 2 were received by animals with visible leg defects, 3 points - average condition, 4 and 5 - good and excellent, respectively. Preliminary data analysis showed the imbalance of the available data. Imbalanced classes are a common problem in machine learning classification where there is a disproportionate ratio of observations in each class. Since most ML algorithms work best when the number of samples in each class are about equal, a balancing procedure was applied. After the preliminary analysis, the year of birth and gender were excluded as predictors of the least importance.

\section{Methods}

4 The algorithms used for this work were selected from a wide range of probabilistic and non-probabilistic methods in order to cover the entire spectrum of existing tools. In addition to the algorithms presented in our work, we tested other methods, for example, the XGboost algorithm, which is commonly used for 
87 solving classification problems, but in our case, it only gave mediocre results. In agricultural mathematics,

88 from the beginning of the 20th century to this day, the statistical methods of Fisher and Wright have been

89 widely used. With the successful use of ML algorithms in various fields of human activity, it was inevitable

90 they have appeared in agricultural problem-solving.

91

92 Classification models were constructed and analyzed using the following ML methods: Random Forest 93 (RF) [16], K-Nearest Neighbors (K-NN) [17,18], artificial neural networks (Neural Networks) [19], C50Tree,

94 Support Vector Machines (SVM) [20], Naive Bayes (NB) [21], GLM [22], Boost [23] and Linear Discriminant

95 Analysis (LDA) [24]. All calculations and simulations were performed in R (version 3.6.1, [25]) using the 96 caret packages [26], DMwR [27]. Leg scores were used as the response variables.

97

\section{K-Nearest Neighbors (K-NN)}

The K-NN classifier is based on the compactness hypothesis, which assumes that a test object will have the same class label as the training objects in the local area of its immediate environment. When the value of $\mathrm{K}$

101 is one, the analyzed object is assigned to a certain class depending on information about its single nearest neighbor. When $\mathrm{K}>1$, every object is assigned to the prevailing class of nearest neighbors. Any clustering algorithm can be considered effective if the compact hypothesis is satisfied, meaning that there exists a partition of objects into groups that the distances between objects from the same group (intra-cluster distances) will be less than a certain value $\varepsilon>0$, and the distance between objects from different groups

106 (cross-cluster distances) is more than $\varepsilon$ [28].

108 Linear Discriminant Analysis (LDA)

109 LDA is a multidimensional analysis section that allows one to evaluate differences between two or more

110 groups of objects using several variables. It is a generalization of Fisher's linear discriminant, a method

111 used in machine learning to find a linear combination of features which characterizes or separates two or 112 more classes of objects or events. The resulting combination can be used as a linear classifier or, more often, 
113 to reduce the dimension before subsequent classification. LDA is closely related to the analysis of variance

114 (ANOVA) procedure. The LDA implements two closely related statistical procedures:

115 1. Interpretation of group differences, needing to answer the question: how a well-used set of variables 116 can form a dividing surface for objects of the training sample and which of these variables are the most 117 informative.

118 2. Classification, i.e. prediction of the value of the grouping factor for the examined group of 119 observations.

121 The support vector machines (SVM)

122 SVM, previously called the "generalized portrait" algorithm, was developed by Soviet mathematicians 123 Vapnik and Chervonenkis [29] and has since gained widespread popularity. The main idea of the classifier 124 on support vectors is to build a separating surface using only a small subset of points lying in the zone 125 critical for separation, while the rest of the correctly classified observations of the training sample outside 126 of this zone are ignored (more precisely, they are a "reservoir" for an optimization algorithm). If there are 127 two classes of observations and a linear form of the boundary between the classes is assumed, then two 128 cases are possible. The first of them relates to the possibility of perfect data separation with the help of 129 some hyperplane. Since there can be many such hyperplanes, the dividing surface is optimal, which is as 130 far as possible from the training points, i.e. having a maximum gap $M$ (margin).

132 Naive Bayes classifier (NB)

133 Naive Bayes classifiers are a family of simple probabilistic ML classifiers based on the application of Bayes

134 theorem. Making the "naive" assumption that all the signs describing the classified objects are completely

135 equal and are not related to each other, then the probability of an object to belong to a given class given its 136 observed features, $P$ (class|features), is calculated using the Bayes formula from known distributions $137 P$ (features I class). The NB assigns the objects to refer to the class that has the greatest probability. 
Neural Networks

140 Neural network models that were born in the process of developing the concept of artificial intelligence

141 have two completely transparent analogies - the biological neural system of the brain and the computer

142 network. Their main paradigm is that the solution in the network is formed by many simple neuron-like

143 elements that form a graph with weighted synaptic (informational) connections that work together and

144 purposefully to obtain a common result. To train artificial neural networks in the R environment, the nnet

145 package [30] was used; it provides flexible functionality for constructing classification models based on a

146 multilayer perceptron.

147

148 GLM

149 Logistic regression is commonly used as a binary classifier for alternate response samples. However, this

150 method can also be generalized to the case with several classes. Nominal or ordinal variables can be used

151 as the simulated response $\mathrm{Y}$, and in both cases, a multidimensional binomial distribution is assumed.

152 Simply put, linear regression should be used to predict a quantitative (i.e., numerical) response variable,

153 and logical regression should be used to predict a qualitative (i.e., categorical) response variable. Both linear

154 regression and logistic regression are types of generalized linear models (GLM).

155

156 Gradient Boosting

157 One of the methods for improving predictions is boosting, which is an iterative process of sequentially

158 constructing private models. Each new model is trained using the information on errors made at the

159 previous stage, and the resulting function is a linear combination of all, considering the minimization of

160 any penalty function. Like bagging, boosting is a general approach that can be applied to many statistical

161 classification methods. The idea of increasing the gradient arose as a result of Leo Braiman's observations

162 that increasing the gradient can be interpreted as an optimization algorithm on an appropriate cost

163 function. Several algorithms for increasing the gradient of direct regression were developed [31][32]. The 
164 Draper et al. [32] approach optimizes the cost function with respect to the functional space by iteratively

165 choosing a function, indicating the direction of the negative gradient.

166

167 The C 50Tree method

168 This method is based on the application of a strategy of dividing data into smaller and smaller parts to

169 identify patterns that can ultimately be used for forecasting. The model itself includes many logical

170 decisions, with decision nodes. They are divided into branches that indicate the choice of solution. The tree

171 ends with leaf nodes (also called terminal nodes), which indicates the result of a combination of decisions.

172 The data to be classified begins at the root node, where the ripple is transmitted to them, and various

173 decisions in the tree, in accordance with the values of the predictors, depending on their influence on the

174 response variable.

175

176 Random Forest

177 Random Forest is a controlled learning method in which the target class is a priori known, and a model is

178 built (classification or regression) to predict future responses. Several hundred decision trees are built for

179 training bootstrap samples. However, at each iteration of the tree construction, randomly selected $m$ from

$180 p$ predictors to be considered, and the partition can be performed on only one of these $m$ variables. The

181 meaning of this procedure, which turned out to be very effective for improving the quality of the obtained

182 solutions, is that with the probability $(p-m) / p$ some potentially dominant predictor that seeks to enter

183 every tree is blocked. By blocking dominants, other predictors will get their chance, and tree variation will

184 increase.

185

186 Data Preparation

187 The number of observations for training models allows one to achieve high predictive effectiveness. The

188 data includes both continuous and high-quality variables, which allows facile problem-solving. The 189 response variable (target variable) was a leg score, which varies from 1 to 5 . For practical reasons, the values 
190 were adjusted and divided into two bins: scores [1:2] - animals with "bad" legs (Q1) and scores [3:5] -

191 animals with "good legs" (Q2). Accuracy was calculated as the proportion of correct predictions of the

192 algorithm, precision, recall, and the F1 score (a harmonic mean of precision and recall). The data points

193 were assigned to the bins (2708 (4930) for Q1; 21876 (19654) for Q2), corresponding to 11\% (20\%) and 89\%

$194(80 \%)$ of measurements for the two breeds. The imbalance of the data classes (a large difference between

195 the numbers of samples in different bins) can negatively affect both the learning and prediction phases of

196 the approach. If the unbalance ratio is high, the decision function favors the "majority" class, where the

197 largest number of samples is located. Not all ML models are affected by unbalanced classes and most

198 probabilistic models are weakly dependent on them. However, problems arise when non-probabilistic

199 classifiers are used. For example, in logistic regression, neural networks, as well as in SVM algorithms, class

200 balance strongly affects their parameters. In the decision trees, random forest, and gradient boosting

201 approaches, class imbalances affect the measures of leaf impurity. To solve the problem of class imbalance,

202 the oversampling method was used. The advantage of using this method is that it does not lead to

203 information loss. Therefore, the leg score data for the bins were balanced using the ROSE package.

204

205

Data Analysis

206 Before choosing the most important predictors and training the prognostic model, a descriptive study of

207 variables was conducted. This process allows for a better understanding of what information each variable

208 contains, as well as to identify possible errors. The procedure for collecting information on farms is

209 determined by the human factor as are other production features of the industry. It is not always possible

210 to enforce measurement collection protocol compliance and obtain data in its entirety; therefore, missing

211 values occur in our dataset. To fill in the missing values, we used the preProcess function from the caret

212 package in R (classification and regression training, http://topepo.github.io/caret/index.html, bagimput

213 method). This method constructs a "bagging" model for each of the available variables based on

214 regression trees, using all other variables as predictors; it requires significant computation time, especially

215 when working with large data sets [23].

Peer) reviewing PDF | (2019:11:43093:1:2:NEW 5 Feb 2020) 
217 Studying the distribution of the response variable relative to quantitative (Muscle Thickness, Back Fat,

218 Average Daily Gain) and qualitative (Breed) variables is an important exercise. Analysis of quantitative

219 variables showed a pronounced asymmetric distribution of some predictors (Back Fat). The calculation of 220 correlations between continuous predictors indicates that they do not contain redundant information 221 (Figure 2).

223 For a predictive model to be useful, it must have a success rate higher than expected by chance or at a 224 certain base level. In classification problems, the base level is the level obtained if all observations are 225 assigned to the majority class. In our case, since $89 \%$ (80\%) of the animals have healthy front (hind) legs, 226 then the expected success rate is $89 \%(80 \%)$ for unbalanced and $50 \%$ for balanced data participating in the 227 training set. Our goal to design predictive models that have a better success rate than the expected one. 228 Since the aim of the study is to assess the state (conformation) of legs by means of selected predictors (e.g. growth and meat quality), we are interested in the proportion of the animals with healthy legs (correspondence with other leg conformation classes is less important). By analyzing the data in this way, one can begin to extract ideas about which variables are most associated with "good" legs. To study the importance of predictors, we also used the Random Forest package. All studies algorithms have identified that the most important predictors are Muscle Thickness, Back Fat, Average Daily Gain, while the predictor

234 Breed is not significant (Figure 3).

Model training

Figure 2 shows that the measurements follow bell-shaped distributions. Therefore, a standardization of the data was carried out by subtracting the mean and dividing each predictor by its standard deviation, so the data obeys the standard normal distribution. 
242 the final assessment, that we designate for an independent trial. The independent test dataset was not used

243 to build a model, it was only used to test the models (results are shown in Fig. 1 as the indTest). The

244 remaining $90 \%$ of samples were randomly divided into $70 \%$ for training and $30 \%$ for testing; the process

245 was repeated 100 times. In every 100 training iterations, hyperparameters were selected using a search 246 within the 10-fold cross-validation structure on a random $70 \%$ subset of the training set. The selected 247 hyperparameters were used to train each ML model on a training set and were tested on a test set in each

248 iteration. All processes were implemented in R. The performance of the final model has been evaluated on

249 the test and on the indTest sets.

250 Performance assessment

251 Model fit and ranking between models is assessed using several scores that can be computed from

252 the number of true positive (TP), true negative (TN), false positive (FP), and false-negative (FN) predictions.

253 These numbers can be applied per class or be aggregated for the entire dataset.

254 Accuracy measures a fraction of correct predictions as is usually represented as a percentage.

$$
\text { Accuracy }=\frac{T P+T N}{T P+T N+F P+F N}
$$

Error rate measures a fraction of incorrectly classified samples.

$$
\text { Error }=\frac{F P+F N}{T P+T N+F P+F N}=1-\text { Accuracy }
$$

Specificity (Precision) is the fraction of correct predictions.

$$
\text { Precision }=\text { Specificity }=\frac{T P}{T P+F P}
$$

Sensitivity (Recall) measures a fraction of correct predictions per the true number of samples.

The F-Measure (F1) is a goodness of fit assessment for a classification analysis that balances

264 precision and recall, ranging between 0 and 1.

$$
\text { Recall }=\text { Sensitivity }=\frac{T P}{T P+F N}
$$

\section{3}

$$
F 1=2 \frac{\text { Precision } \times \text { Recall }}{\text { Precision }+ \text { Recall }}
$$



qualitative observations. Let $p_{e}$ be the expected number of agreements and $p_{o}$ be the observed number of agreements.

$$
\kappa=\frac{p_{o}-p_{e}}{1-p_{e}}
$$

\section{Results}

272 The ML approach was able to predict the state of the front and hind legs. The Random Forest method surpassed all other learning algorithms in all tested scenarios. The KNN approach was a close runner-up (Table 2). The superiority of RF and $\mathrm{KNN}$ is due to a lower value of the variance of forecasting indicators as compared to SVM and NB approaches. SVM and NB were among the least effective forecasting methods in this study, providing the lowest correlation and the largest forecasting errors. A graphical interpretation (Figure 1) of the comparative analysis of the predicted value of all models shows that all models in the training set receive more accurate forecasts than in the test set. In order to address the overfitting issue, we have replaced the "KNN" method by the "KKNN" method. KKNN method is more flexible and permits selection of the measure of similarity, the shape of the kernel function, as well as the estimation of the optimal value of the parameter $k$ using the cross-validation approach. In the case of the Random Forest algorithm, varying parameters of cross-validation, selection of the optimal cut-off value, the accuracy can resolved.

Both RF and KNN models provide higher accuracy of prediction compared to other models. To determine what models achieved the best results in solving the problem after the training procedures and their optimization, a comparative analysis was carried out. Obviously, the indicators obtained by validation are estimates of the ability of the model to predict new observations and these estimates have deviations. A comparison was made between all models with the non-parametric Friedman test and a pairwise comparison of all models, the results of which are summarized in Table 3. The best predictive capabilities

\section{Discussion}


295 The increase in the prevalence of leg weakness in pigs in the middle of the 20th century coincided with a

296 surge of targeted breeding work to increase the growth rate of animals. This was mainly due to economic 297 pressure and the need to shorten the period from birth to slaughter. Since in wild boars, requiring about 298 two years to reach maturity, osteochondrosis is not observed, it was proposed that there was a relationship 299 between the growth qualities and weakness of the legs. Several large population studies have shown a 300 positive correlation between these traits [1,11,12]. Lundeheim [13] noted that pigs with clinical signs of leg

301

302

303

304

305

306

307

\section{Conclusions}

316 Leg weakness is a source of significant economic loss in pig production, therefore, the search for predictors 317 of leg condition is of great interest and potential value. Machine learning is a relatively new paradigm in 318 computational biology. The problem of processing and comprehending a huge data stream poses a 319 challenge for researchers to develop new computational methodologies. In our opinion and experience, 320 ML algorithms are a good alternative to parametric models to solve many problems in biology. ML 321 focuses on algorithmically constructed models with optimal forecasting as their ultimate goal. One of the 322 important additions to the accurate forecasting of ML is the ability to obtain data for training from empirical 323 observations and use ML to train algorithms for recognizing phenomena that may be overlooked. Our 324 comparison of various machine learning algorithms proved that growth rate and meat parameters were 
325 effective predictors of the condition of pig legs. PigLeg provides a powerful tool to assess the health of

326 the animals. The best predictive performance was achieved by the Random Forest approach.

327

328 Supplementary Materials: The scripts and raw data can be found at http://www.compubioverne.group/data-and329 software/.

330 Acknowledgments:

331 Author Contributions: Conceptualization, S.B. and L.G.; methodology, S.B. and T.T.; software, S.B.; validation, S.B.;

332 formal analysis, S.B.; investigation, M.K., L.G, and O.K.; resources M.K. and O.K.; data curation, M.K, and L.G.;

333 writing-original draft preparation, S.B. and T.T; writing-review and editing, T.T and D.C.; visualization, S.B.;

334 supervision, S.B.; project administration, L.G.; funding acquisition, SB, LG, MK, and OK.

335 Funding: SB, LG, MK, and OK were supported by the Russian Foundation for Basic Research grant 19-016-00068 A.

336 Conflicts of Interest: The authors declare no conflict of interest.

337 Abbreviations

$\begin{array}{ll}\text { NGS } & \text { Next-Generation Sequencing } \\ \text { QTL } & \text { Quantitative Trait Locus } \\ \text { GWAS } & \text { Genome-Wide Association Studies } \\ \text { ML } & \text { Machine Learning } \\ \text { ADG } & \text { Average Daily Gain } \\ \text { MT } & \text { Muscle thickness } \\ \text { BF } & \text { Back Fat } \\ \text { RF } & \text { Random Forest } \\ \text { KNN } & \text { K-Nearest Neighbors } \\ \text { NN } & \text { Neural Networks } \\ \text { SVM } & \text { Support Vector Machines } \\ \text { NB } & \text { Naïve Bayes } \\ \text { GLM } & \text { Generalized Linear Models } \\ \text { LDA } & \text { Linear Discriminant Analysis }\end{array}$

\section{References}

339 1. Ekman, S.; Carlson, C.S. The pathophysiology of osteochondrosis. Vet. Clin. North Am. Small Anim. 
Pract. 1998, 28, 17-32.

341 2. Le, T.H.; Christensen, O.F.; Nielsen, B.; Sahana, G. Genome-wide association study for conformation 342 traits in three Danish pig breeds. Genet. Sel. Evol. 2017, 49, 12.

343 3. Aasmundstad, T.; Olsen, D.; Sehested, E.; Vangen, O. The genetic relationships between conformation assessment of gilts and sow production and longevity. Livest. Sci. 2014, 167, 33-40.

345 4. Knauer, M.T.; Cassady, J.P.; Newcom, D.W.; See, M.T. Phenotypic and genetic correlations between 346 gilt estrus, puberty, growth, composition, and structural conformation traits with first-litter 347 reproductive measures. J. Anim. Sci. 2011, 89, 935-942.

348 5. Morota, G.; Ventura, R.V.; Silva, F.F.; Koyama, M.; Fernando, S.C. BIG DATA ANALYTICS AND 349 PRECISION ANIMAL AGRICULTURE SYMPOSIUM: Machine learning and data mining advance 350 predictive big data analysis in precision animal agriculture1. Journal of Animal Science 2018, 96, 1540$351 \quad 1550$.

352 6. Putz, A.M.; Harding, J.C.S.; Dyck, M.K.; Fortin, F.; Plastow, G.S.; Dekkers, J.C.M.; PigGen Canada 353 Novel Resilience Phenotypes Using Feed Intake Data From a Natural Disease Challenge Model in 354 Wean-to-Finish Pigs. Front. Genet. 2018, 9, 660.

355 7. Howard, J.T. The use of "Big Data" in a modern swine breeding program now and in the future.

356 8. Shahinfar, S.; Kahn, L. Machine learning approaches for early prediction of adult wool growth and 357 quality in Australian Merino sheep. Comput. Electron. Agric. 2018, 148, 72-81.

358 9. Shahinfar, S.; Kelman, K.; Kahn, L. Prediction of sheep carcass traits from early-life records using 359 machine learning. Comput. Electron. Agric. 2019, 156, 159-177.

360 10. Gorczyca, M.T.; Milan, H.F.M.; Maia, A.S.C.; Gebremedhin, K.G. Machine learning algorithms to 361 predict core, skin, and hair-coat temperatures of piglets. Comput. Electron. Agric. 2018, 151, 286-294.

362 11. Breiman, L. Random Forests. Mach. Learn. 2001, 45, 5-32.

363 12. Cover, T.; Hart, P. Nearest neighbor pattern classification. IEEE Transactions on Information Theory $364 \quad 1967,13,21-27$.

365 13. Lantz, B. Machine Learning with R: Expert techniques for predictive modeling to solve all your data analysis 
366 problems; Packt Publishing Ltd, 2015; ISBN 9781784394523.

367 14. Ripley, B.D.; Hjort, N.L. Pattern Recognition and Neural Networks; Cambridge University Press, 1996; $368 \quad$ ISBN 9780521460866.

369 15. Cortes, C.; Vapnik, V. Support-vector networks. Mach. Learn. 1995, 20, 273-297.

370 16. Rennie, J.D.; Shih, L.; Teevan, J.; Karger, D.R. Tackling the poor assumptions of naive Bayes text 371 classifiers. In Proceedings of the Proceedings of the 20th international conference on machine learning 372 (ICML-03); 2003; pp. 616-623.

373 17. Walker, S.H.; Duncan, D.B. Estimation of the probability of an event as a function of several 374 independent variables. Biometrika 1967, 54, 167-179.

375 18. Breiman, L. Arcing classifier (with discussion and a rejoinder by the author). The Annals of Statistics $376 \quad 1998,26,801-849$.

377 19. Fisher, R.A. THE USE OF MULTIPLE MEASUREMENTS IN TAXONOMIC PROBLEMS. Annals of $378 \quad$ Eugenics 1936, 7, 179-188.

379 20. Team, R.C.; Others R: A language and environment for statistical computing. 2013.

380 21. Kuhn, M.; Others Building predictive models in R using the caret package. J. Stat. Softw. 2008, 28, 138126

382 22. Torgo, L. Data mining with R: learning with case studies; Chapman and Hall/CRC, 2011.

383 23. Shitikov, V.K.; Mastitsky, S.E. Classification, regression, Data Mining algorithms using R 2017.

384 24. Vapnik, V.; Chervonenkis, A. Theory of pattern recognition 1974.

385 25. Ripley, B.; Venables, W. nnet: Feed-forward neural networks and multinomial log-linear models. $R$ $386 \quad$ package version 2011, 7.

387 26. Friedman, J.H. Greedy function approximation: a gradient boosting machine. Department of 388 Statistics. University of Stanford: Stanford, CA, USA 1999.

389 27. Mason, L.; Baxter, J.; Bartlett, P.L.; Frean, M.R. Boosting Algorithms as Gradient Descent. In Advances 390 in Neural Information Processing Systems 12; Solla, S.A., Leen, T.K., Müller, K., Eds.; MIT Press, 2000; $391 \quad$ pp. 512-518. 
392 28. Jørgensen, B.; Andersen, S. Genetic parameters for osteochondrosis in Danish Landrace and Yorkshire 393 boars and correlations with leg weakness and production traits. Anim. Sci. 2000, 71, 427-434.

394 29. Nakano, T.; Brennan, J.J.; Aherne, F.X. LEG WEAKNESS AND OSTEOCHONDROSIS IN SWINE: A 395 REVIEW. Can. J. Anim. Sci. 1987, 67, 883-901.

396 30. Lundeheim, N. Genetic Analysis of Osteochondrosis and Leg Weakness in the Swedish Pig Progeny 397 Testing Scheme. Acta Agriculturae Scandinavica 1987, 37, 159-173.

398 31. Van der Wal, P.G.; Van der Valk, P.C.; Goedegebuure, S.A.; Van Essen, G. Osteochondrosis in six 399 breeds of slaughter pigs: II. Data concerning carcass characteristics in relation to osteochondrosis. Vet. $400 \quad$ Q. 1980, 2, 42-47.

401 32. Draper, D.D.; Rothschild, M.F.; Christian, L.L. Effects of divergent selection for leg weakness on 402 muscle and bone characteristics in Duroc swine. Genet. Sel. Evol. 1992, 24, 363. 


\section{Figure 1}

Accuracy for training and testing sets for different ML approaches.

A graphical interpretation of the comparative analysis of the predicted value of all models shows that all models in the training set receive more accurate forecasts than in the test set. At the same time, the RF and KNN models provide high accuracy of prediction relative to other models. To determine the models that achieve the best results in solving the problem after the training procedures and their optimization, a comparative analysis was carried out. Obviously, the indicators obtained by validation are estimates of the ability of the model to predict new observations and these estimates have deviations.

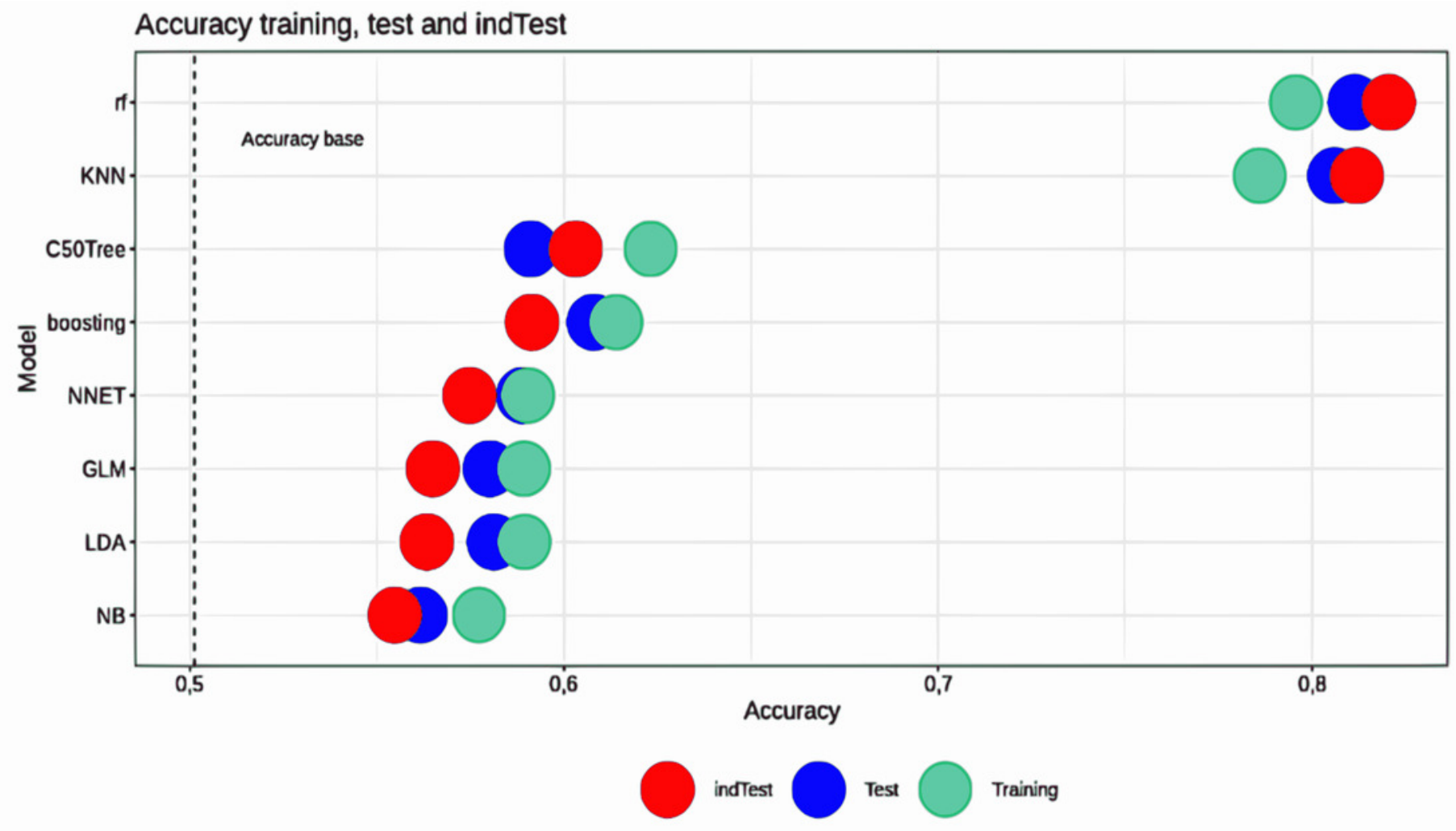




\section{Figure 2}

Analysis of the collected measurements.

Variables (Average Daily Gain (A, B), Back Fat (C, D), and Muscle Thickness (E, F)). Back Fat (C) has an asymmetric distribution. Concordance analysis of predictors (G) shows a moderate correlation between the three parameters. This indicates that they do not contain redundant information.

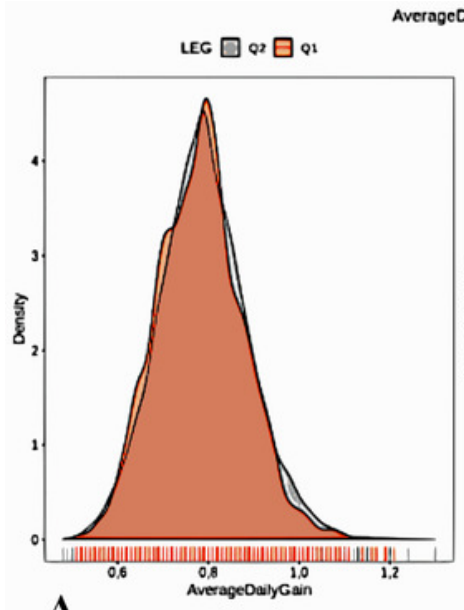

A

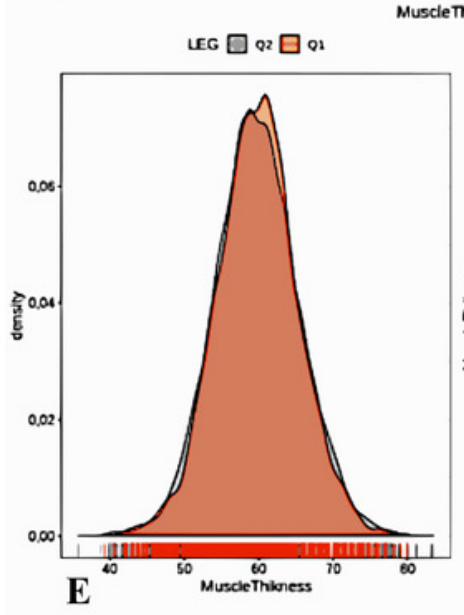

LEG 白 Q2 白 Q

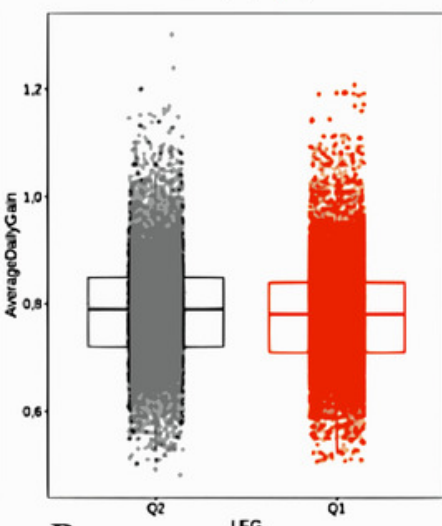

B

ness

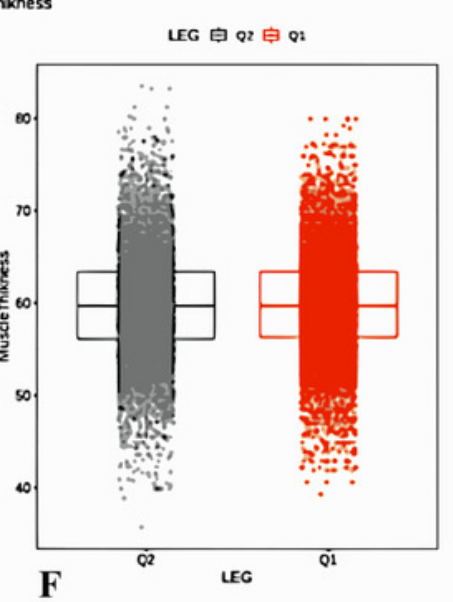

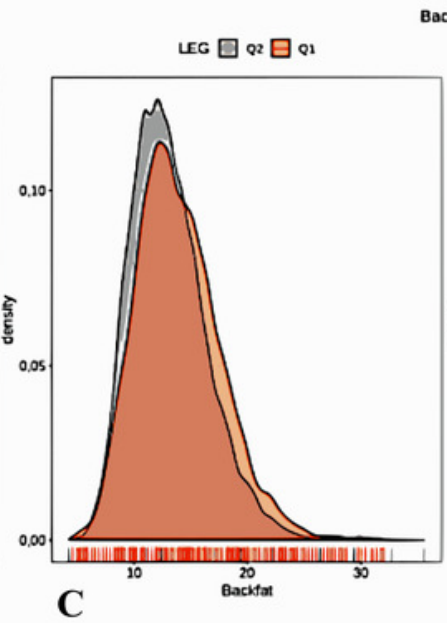

Backlat
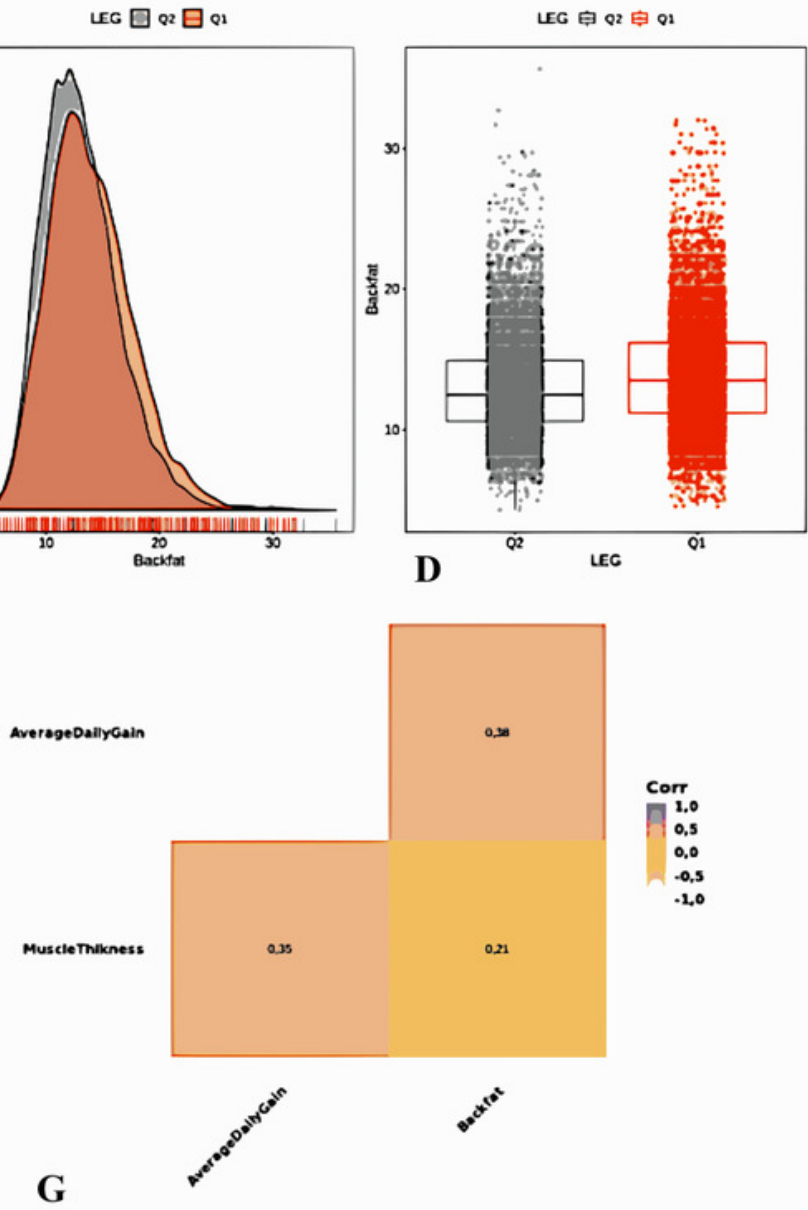


\section{Figure 3}

Relative importance of leg weakness predictors as assessed by Accuracy (A) and Gini (B).

Since the aim of the study is to assess the state (conformation) of legs by means of selected predictors (growth and meat quality), each variable is analyzed with respect to the variable Q2 = "good". By analyzing the data in this way, one can begin to extract ideas about which variables are most associated with "good" legs. Alternatively, to study the importance of predictors, we use the Random Forest package. All studies algorithms have identified that the most important predictors are Muscle Thickness, Back Fat, Average Daily Gain, while the predictor Breed is not significant.
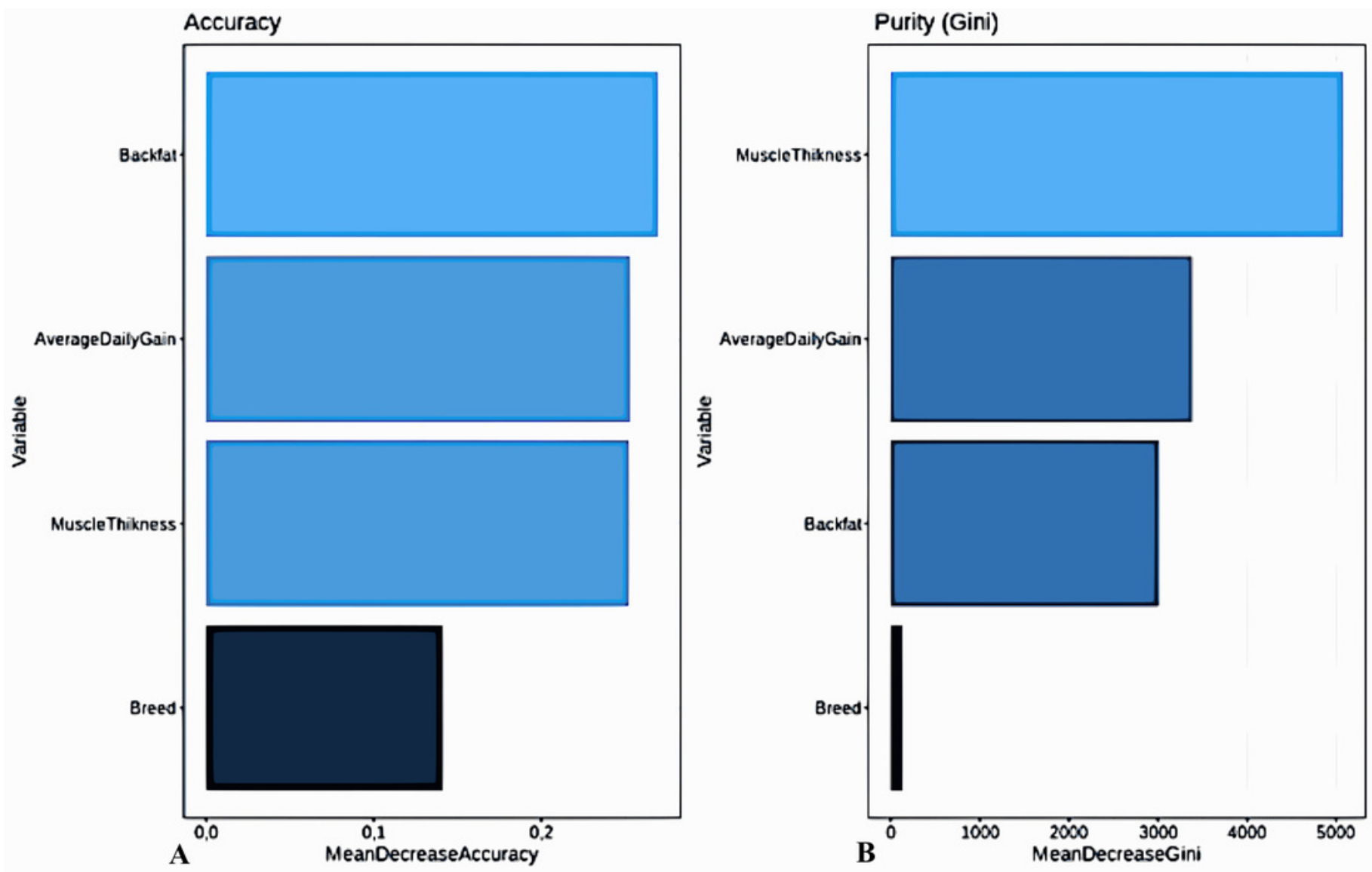


\section{Table $\mathbf{1}$ (on next page)}

Sample description.

The dataset contains 21,247 females and 3337 males. 12,195 of Landrace and 12389 of Large White breeds. Predictors: Average Daily Gain, Backfat Thickness, Muscle, Thickness, Birth Date, Breed, Sex. Dependent variables: scores for front and back legs. 
1

\begin{tabular}{lllllll}
\hline Variable & $\mathbf{m i n}$ & $\mathbf{1}^{\text {st }} \mathbf{Q u}$. & median & mean & $\mathbf{3}^{\text {rd }} \mathbf{Q u .}$ & max \\
\hline Average Daily Gain & 0.33 & 0.72 & 0.79 & 0.79 & 0.85 & 1.61 \\
Backfat Thickness & 4.30 & 10.90 & 12.90 & 13.25 & 15.20 & 35.60 \\
$\begin{array}{l}\text { Muscle Thickness } \\
\text { Birth Date }\end{array}$ & 32.12 & 56.04 & 59.70 & 59.68 & 63.40 & 96.00 \\
$\begin{array}{l}\text { Scores } \\
\text { Front Legs }\end{array}$ & 2012 & 2014 & 2015 & 2015 & 2016 & 2016 \\
Back Legs & & & & & & \\
\hline
\end{tabular}

2 


\section{Table 2 (on next page)}

Comparison between the models using the testing dataset.

ML models were able to predict the state of the fore and hind legs. RF surpassed all other learning algorithms in all respects and scenarios. In some cases, RF did not have significant superiority over KNN. Accordingly, KNN was the second most efficient algorithm among all the characteristics and scenarios. 


\begin{tabular}{|l|r|r|r|r|r|}
\hline Model & \multicolumn{1}{|l|}{ Accuracy } & \multicolumn{1}{|l|}{ Kappa } & \multicolumn{1}{l|}{ P-value $\kappa$} & \multicolumn{1}{l|}{ Sensitivity } & \multicolumn{1}{l|}{ Specificity } \\
\hline RF & 0.8846 & 0.7693 & $<2.2 \mathrm{e}-16$ & 0.8232 & 0.9463 \\
\hline KNN & 0.8754 & 0.7509 & $3.238 \mathrm{e}-16$ & 0.8013 & 0.9499 \\
\hline C50Tree & 0.6469 & 0.294 & 0.001603 & 0.5746 & 0.7195 \\
\hline Boost & 0.6035 & 0.207 & 0.09968 & 0.5995 & 0.6075 \\
\hline NNET & 0.5667 & 0.1335 & 0.01852 & 0.5619 & 0.5716 \\
\hline LDA & 0.563 & 0.1258 & $2.343 \mathrm{e}-05$ & 0.5986 & 0.5272 \\
\hline GLM & 0.5624 & 0.1246 & $5.043 \mathrm{e}-05$ & 0.5971 & 0.5275 \\
\hline SVM & 0.5603 & 0.1202 & $3.248 \mathrm{e}-05$ & 0.653 & 0.4671 \\
\hline NB & 0.5411 & 0.0816 & $<2.2 \mathrm{e}-16$ & 0.6984 & 0.3832 \\
\hline
\end{tabular}




\section{Table 3 (on next page)}

Search results among all the models of non-parametric tests of Friedman and paired comparison of all models.

A comparison was made between all models with the non-parametric Friedman test and a pairwise comparison of all models. The best predictive capabilities in the dataset were shown by the Random Forest approach. In addition, it must be noted that such signs as Muscle Thickness, Back Fat, Average Daily Gain can act as predictors of leg weakness. Information on breed and gender were not significant for assessment the status of legs. 


\begin{tabular}{llrlll}
\hline Model A & Model B & p-value & Model A & Model B & p-value \\
\hline boosting & arbol & $4.37 \mathrm{E}-02$ & NB & logistic & $2.17 \mathrm{E}-08$ \\
KNN & arbol & $2.17 \mathrm{E}-08$ & NET & arbol & $2.17 \mathrm{E}-08$ \\
KNN & boosting & $2.17 \mathrm{E}-08$ & NET & boosting & $2.17 \mathrm{E}-08$ \\
LDA & arbol & $2.17 \mathrm{E}-08$ & NET & KNN & $2.17 \mathrm{E}-08$ \\
LDA & boosting & $2.17 \mathrm{E}-08$ & NET & LDA & $1.31 \mathrm{E}-07$ \\
LDA & KNN & $2.17 \mathrm{E}-08$ & NET & logistic & $1.31 \mathrm{E}-07$ \\
logistic & arbol & $2.17 \mathrm{E}-08$ & NET & NB & $2.17 \mathrm{E}-08$ \\
logistic & boosting & $2.17 \mathrm{E}-08$ & rf & arbol & $2.17 \mathrm{E}-08$ \\
logistic & KNN & $2.17 \mathrm{E}-08$ & rf & boosting & $2.17 \mathrm{E}-08$ \\
logistic & LDA & $9.30 \mathrm{E}-02$ & $\mathrm{rf}$ & KNN & $2.17 \mathrm{E}-08$ \\
NB & arbol & $2.17 \mathrm{E}-08$ & $\mathrm{rf}$ & LDA & $2.17 \mathrm{E}-08$ \\
NB & boosting & $2.17 \mathrm{E}-08$ & $\mathrm{rf}$ & logistic & $2.17 \mathrm{E}-08$ \\
NB & KNN & $2.17 \mathrm{E}-08$ & $\mathrm{rf}$ & NB & $2.17 \mathrm{E}-08$ \\
NB & LDA & $2.17 \mathrm{E}-08$ & $\mathrm{rf}$ & NET & $2.17 \mathrm{E}-08$ \\
\hline
\end{tabular}

Friedman rank sum test

Friedman chi-squared $=286.85, \mathrm{df}=6, \mathrm{p}$-value $<2.2 \mathrm{e}-16$ 